\title{
Liste des rapporteurs en 2013 et 2014
}

\section{(2) OpenEdition}

1 Journals

Édition électronique

URL : https://journals.openedition.org/travailemploi/6603

DOI : 10.4000/travailemploi.6603

ISSN : 1775-416X

\section{Éditeur}

DARES - Ministère du Travail

\section{Édition imprimée}

Date de publication : 1 janvier 2015

ISSN : 0224-4365

\section{Référence électronique}

"Liste des rapporteurs en 2013 et 2014 », Travail et Emploi [En ligne], 141 I janvier-mars 2015, mis en ligne le 01 janvier 2015, consulté le 04 juillet 2022. URL : http://journals.openedition.org/travailemploi/ 6603 ; DOI : https://doi.org/10.4000/travailemploi.6603 


\section{La revue remercie les rapporteurs qui ont contribué à l'évaluation des projets d'article soumis en 2013 et 2014}

Sarah ABDELNOUR
Élisabeth ALGAVA
Anne-Marie ARBORIO
Christelle AVRIL ${ }^{*}$ )
Samia BADJI
François BAFOIL
Olivier BAGUELIN
Muriel BARLET
Isabelle BASZANGER
Melika BEN SALEM
Antoine BEVORT
Sylvie BLASCO
Géraldine BLOY
Isabel BONI-LE GOFF
Annie BORZEIX
Gerhard BOSCH
Stephen BOUQUIN
Thomas BRODATY
Cécile BROUSSE
Anne-Sophie BRUNO
Jean-Paul CADET
José CALDERON
Patrick CASTEL
Sylvie CÉLÉRIER
Sébastien CHAUVIN
Matthieu CLÉMENT
Valérie COHEN
Pierre CONCIALDI
Bernard CONTER
Delphine CORTEEL
Christel COTON
Alain COTTEREAU
Olivier COUSIN
Thomas COUTROT
Aï-Thu DANG
Fanny DARBUS
Isabelle DAUGAREILH
Jacques DEFRANCE
Didier DEMAZIÈRE
Pascal DEPOORTER
Cécile DÉTANG-DESSENDRE
Claude DIDRY

Sophie DIVAY
Nicolas DODIER
Vincent DUBOIS
Richard DUHAUTOIS
Xavier DUNEZAT
Arnaud DUPRAY
Cédric DURAND
Jacky FAYOLLE
Marc FERRACCI
Gaëlle FERRANT
Sébastien FLEURIEL
Anne FRETEL
Charles GADÉA
Bertrand GARBINTI
Bernard GAZIER
Eric GEERKENS
Jean-François GEHANNO
Ariane GHIRARDELLO
Martin GIRAUDEAU
Bernard GOMEL
Dominique GOUX
Agnès GRAMAIN
Mathieu GRÉGOIRE(*)
Michel GROSSETTI
Guy GROUX
Philippe HAMMAN
Anne-Chantal HARDY
Antoine JEAMMAUD
Nicolas JOUNIN
Morgan JOUVENET
Nathalie LAPEYRE
Thomas LE BARBANCHON
Jean-Pierre LE CROM
Amélie LE RENARD
Claire LEMERCIER
Danièle LINHART
Claire MARBOT
Olivier MASCLET
Lilian MATHIEU
Alexandre MATHIEU-FRITZ
Caroline MAZAUD
Christine MENNESSON

\author{
Arnaud MIAS \\ Fanny MIKOL \\ Julian MISCHI \\ Frédéric MOATTY \\ Vincent MOENECLAEY \\ Eva MORENO GALBIS \\ Sandrine NICOURD \\ Alexandra OESER \\ Ariane PAILHÉ \\ Florence PALPACUER \\ Yannis PAPADANIEL \\ Tommaso PARDI \\ Sonia PATY \\ Antoine PELICAND \\ Jérôme PÉLISSE \\ Hélène PÉRIVIER \\ Michel PIALOUX \\ Emmanuel PIERRU \\ Maria-Teresa PIGNONI \\ Marie PLESSZ \\ Patrick POMMIER \\ Sophie PONTHIEUX \\ Maxime QUIJOUX \\ Bertrand RÉAU \\ Véronique RÉMY \\ Nicolas RENAHY \\ Muriel ROGER \\ Patrick ROUSSET \\ Catherine SAUVIAT \\ François-Xavier SCHWEYER \\ Maud SIMONET \\ Anne VEGA \\ Josiane VERO \\ Xavier VIGNA \\ Michel VILLETTE \\ Karel YON \\ Hélène ZAJDELA \\ Philippe ZAMORA \\ Bénédicte ZIMMERMANN \\ Emmanuelle ZOLESIO
}

(*) Pour les évaluations réalisées avant leur entrée au comité de rédaction. 